\title{
BMJ Open Quality ELECTIVIST: a novel system to improve elective caesarean section booking
}

\author{
Katherine Robertson, ${ }^{1}$ Joe Clacey, ${ }^{2}$ Rhiannon D'Arcy, ${ }^{1}$ Neveen Khan, ${ }^{3}$ \\ Aparna Reddy ${ }^{1,4}$
}

To cite: Robertson K, Clacey J, D'Arcy R, et al. ELECTIVIST: a novel system to improve elective caesarean section booking. BMJ Open Quality 2018;7:e000350. doi:10.1136/ bmjoq-2018-000350

- Additional material is published online only. To view please visit the journal online (http://dx.doi.org/10.1136/ bmjoq-2018-000350).

Received 31 January 2018 Revised 25 July 2018 Accepted 31 July 2018
Check for updates

(C) Author(s) (or their employer(s)) 2018. Re-use permitted under CC BY-NC. No commercial re-use. See rights and permissions. Published by BMJ.

${ }^{1}$ Department of Obstetrics and Gynaecology, Buckinghamshire Healthcare NHS Trust, Aylesbury, UK

${ }^{2}$ Mental Health Division, Oxford Health NHS Foundation Trust,

Oxford, Oxfordshire, UK

${ }^{3}$ Department of Obstetrics and Gynaecology, Milton Keynes Hospital NHS Foundation Trust, Milton Keynes, UK

${ }^{4}$ Department of Obstetrics, Oxford University Hospitals NHS Foundation Trust, Oxford, UK

Correspondence to Dr Katherine Robertson; k.e.robertson@gmail.com

\section{ABSTRACT}

Elective caesarean sections (ELCS) vary widely in surgical complexity and are routinely performed between 39 and 40 weeks of pregnancy. Unselected ELCS lists may create clinical risk due to inappropriately complex case mixes and over-running theatre time, impacting on emergency care. Despite evidence that ELCS list over-run is a widespread concern for many units, there is a paucity of literature regarding effective ELCS booking systems.

We designed a novel ELCS risk scoring system, ELECTIVIST, comprising a risk assessment by the booking clinician and allocation of a complexity score to each case from 1 to 6 . The maximum risk score for any one patient was 6 , with a maximum total score on any one ELCS list of 6 and a maximum of three cases per list. We performed a retrospective analysis of all ELCS performed in our unit in 2016 using existing booking information and theatre data to assess existing case mix complexity and theatre over-run. This showed that $36 \%$ of ELCS lists were overbooked with inappropriately complex case mix and $21 \%$ of lists over-ran with $6 \%$ impacting on emergency obstetric theatres. Assessment of the impact of ELECTIVIST on ELCS capacity prior to implementation showed that no additional capacity was required to accommodate existing complexity. At 6 months following implementation, theatre over-run was reduced to $10 \%$ and over-run impacting on emergency theatre to $1 \%$. The requirement for extra ELCS lists to accommodate capacity reduced by $66 \%$.

ELECTIVIST is a novel system that improves ELCS booking using existing capacity and reduces theatre list over-run. It is transferable, cost neutral and could be widely applied in obstetric units.

\section{PROBLEM}

Our unit is a large district general hospital with approximately 5500 deliveries annually. The caesarean section rate in 2016 was $27.2 \%$ and $11 \%$ of all deliveries were performed as planned elective caesarean sections (ELCS).

There are two maternity operating theatres available with separate theatre staff teams for elective and emergency obstetric cases. ELCS lists are scheduled every morning from 0800 to 1300 hour on Mondays to Thursday and on alternate Fridays. There are two consultant-delivered lists per week and the remainder are performed by junior surgeons operating independently on low or moderate risk cases. After 1300 hours, there is one operating theatre available for emergencies. Each
ELCS list has three slots which are booked in antenatal clinic using a master Excel spreadsheet accessed by antenatal clinic midwives. Patient details are entered into the spreadsheet, along with any identified risk factors and the indication for caesarean section.

We identified two main problems with ELCS lists at our Trust. First, the existing ELCS booking process did not involve any selection of what cases were allocated to a particular list. This resulted in unselected ELCS lists, often leading to inappropriately complex case mix on lists carried out by more junior surgeons. Second, unselected ELCS lists of high-complexity cases could over-run beyond the allocated time for list. This resulted in the third ELCS case either being cancelled or performed after 1300 hours in the emergency maternity theatre, creating a clinical risk of a simultaneous obstetric emergency without a free operating theatre.

Our objective was to improve the ELCS booking process by using a novel risk scoring system, ELECTIVIST, to reduce the number of lists with an inappropriately complex case mix. The overall aim was to use existing theatre capacity in a more intelligent way, reduce the need for extra ELCS lists and reduce list over-run, particularly that which impacted on emergency obstetric theatre use. There was no existing information on our case mix complexity and theatre over-run so our objective was to audit this data before and after implementation to assess the impact of ELECTIVIST over a 6-month intervention time period.

\section{BACKGROUND}

Caesarean section is one of the most commonly performed operations worldwide. In the UK in 2016-2017, $27.8 \%$ of women delivered by caesarean section with $15.5 \%$ of women having an ELCS. ${ }^{1}$ Most ELCS are performed between 39 and 40 weeks of gestation and routine elective surgery booking procedures are therefore unsuitable. ELCS cases vary widely in surgical complexity but 
there are little published data regarding the impact of this on surgical time and theatre usage.

One study showed that operative times ranged from 13 to $108 \mathrm{~min}$ and identified previous Caesarean deliveries, increased scar tissue, tubal ligation and surgical experience as factors that increased ELCS operative times. In particular, the number of previous caesarean deliveries increased the mean operative time linearly from $39.5 \mathrm{~min}$ in subjects with no previous caesarean deliveries to $52.8 \mathrm{~min}$ in subjects with three or four previous caesarean deliveries. ${ }^{2}$ Another study showed that total operative time was significantly longer among obese patients compared with women with normal BMI with prolonged operation time of over $60 \mathrm{~min}$ associated with a history of previous caesarean sections, grade of the surgeon and higher estimated blood loss. The authors concluded that there were multiple factors influencing ELCS operative time but some of these factors related to patient characteristics were identifiable early during the preoperative planning period and could be used to plan an appropriate theatre list size. They hypothesised that this could impact ELCS list performance and improve theatre safety and efficiency, while reducing costs and maximising training opportunities. $^{3}$

The Obstetric Anaesthetists' Association and Association of Anaesthetists of Great Britain \& Ireland (OAA/ AAGBI) guidelines for obstetric anaesthetic services recommend that elective caesarean sections should neither interfere with delivery unit work nor be interrupted by emergencies and that such interruptions and delays (to both the scheduled and the emergency workload) should be audited and monitored with necessary action taken to correct recurrence. ${ }^{4}$ However, ELCS list theatre over-run appears to be a common problem for many maternity units with several presented audits at various units describing common themes.

A UK national survey of ELCS availability in 2008 showed that many units are struggling to meet the OAA/ AAGBI recommendations regarding elective surgery provision with $37 \%$ of units frequently performing ELCS outside normal working hours if not done on the scheduled day list and $21 \%$ units cancelling one or more ELCS cases per week. This survey reported 'unit activity' as the main perceived problem with ELCS list efficiency in 63\% of respondents, followed by 'organisational' factors at $47 \% .^{5}$ One audit of ELCS lists in an Australian maternity unit demonstrated other factors contributing to theatre efficiency. Their findings showed that the surgical component of the operating list was $54 \%$ of the total theatre time, with $12 \%$ on anaesthetic time and $34 \%$ on non-surgical and non-anaesthetic activities designated as preparation time. They also found that operator seniority impacted on total case time and cases with a junior operator were more likely to be delayed, with an average over-run of 37 min for an ELCS list performed by a junior operator. The recommendations from these findings suggested improving case mix planning and turnover times between cases. ${ }^{6}$
Despite evidence that ELCS list over-run is a widespread concern for many units, there is a paucity of literature regarding effective ELCS booking systems. A recent quality improvement project presented a four-point categorisation tool which ranked caesareans based on their complexity and calculated the average amount of time required for each category from $\mathrm{A}$ to $\mathrm{D}$. This information was then passed to the booking and scheduling department who could then allocate the appropriate amount of time to each case based on the degree of complexity and likely time needed to complete the procedure. This system reduced over-run from $80 \%$ to $40 \%$ of ELCS lists and demonstrates valuable proof of efficiency improvement when ELCS booking is improved. ${ }^{7}$

\section{BASELINE MEASUREMENT}

We designed a novel ELCS risk scoring system, ELECTIVIST, comprising a risk assessment by the booking clinician who then allocated a complexity score based on this to each case on a scale from 1 to 6 . We designated the maximum risk score for any one patient as 6 and set a standard of a maximum total score on any one ELCS list of 6 and a maximum of three cases per list. Our baseline measurement was a retrospective analysis of all ELCS operations performed in 2016 at our unit.

We collected data on the risk scores for each individual case and the total score per ELCS list from the existing booking Excel spreadsheet. This measurement was to assess our current case mix complexity and to see how many lists had a a score of $>6$ and were therefore overbooked. We collected data on the surgical and theatre time from hospital records to assess whether the risk score correlated with surgical operating time, total theatre time and incidence of over-running with impact on emergency theatre capacity. We defined theatre over-run as surgical time finishing past 1300 hours and theatre over-run impacting on emergency theatres as an ELCS case performed in the emergency maternity theatre after 1300 hours. We also collected data on the number of extra ELCS lists performed to accommodate capacity in 2016 . We then performed a comparative analysis of our actual case complexity and theatre capacity to assess the monthly and annual impact of ELECTIVIST on ELCS capacity prior to implementation. We planned to repeat this analysis at 6 months to evaluate the impact of the new booking form on case mix complexity per list, total theatre time and incidence of over-running.

The results of this baseline measurement are described in figure 1 .

The baseline measurement showed that only $16 \%$ of ELCS lists were of appropriate complexity with $32 \%$ of lists overbooked with inappropriately complex case mix. Theatre over-run occurred in 21\% of ELCS lists and theatre over-run which impacted on emergency theatres occurred in $6 \%$ of ELCS lists. There was widespread variation in the case mix complexity per ELCS list, from 1 to 11 (figure 1B). In 2016, the number of extra ELCS lists performed was 8 
A)

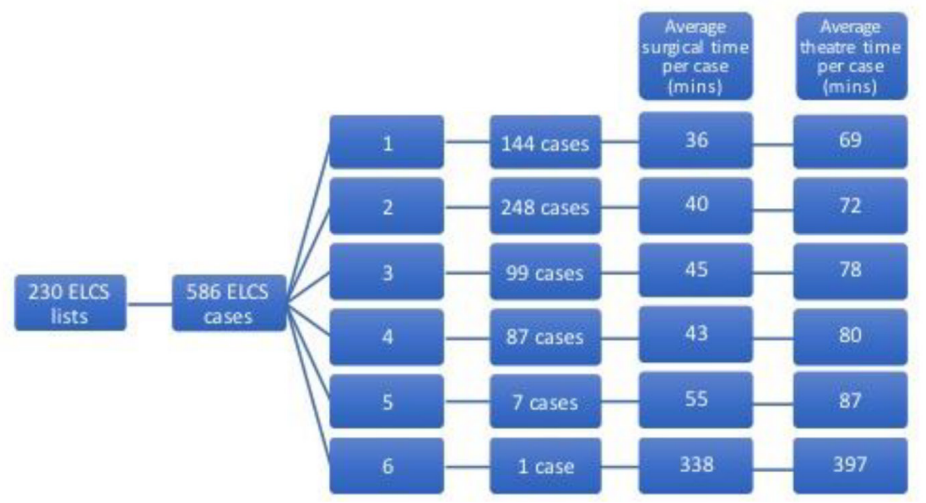

B)

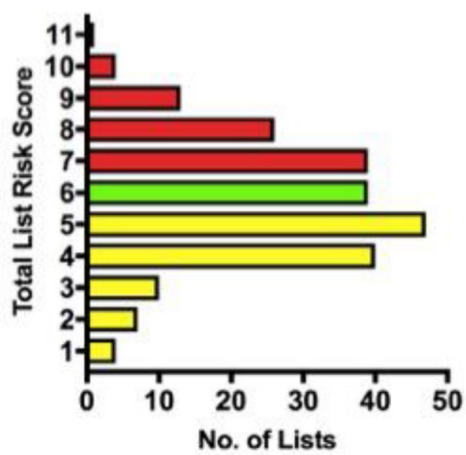

D)

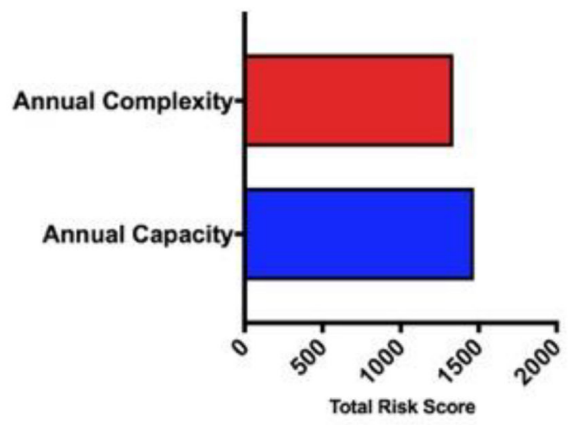

C)

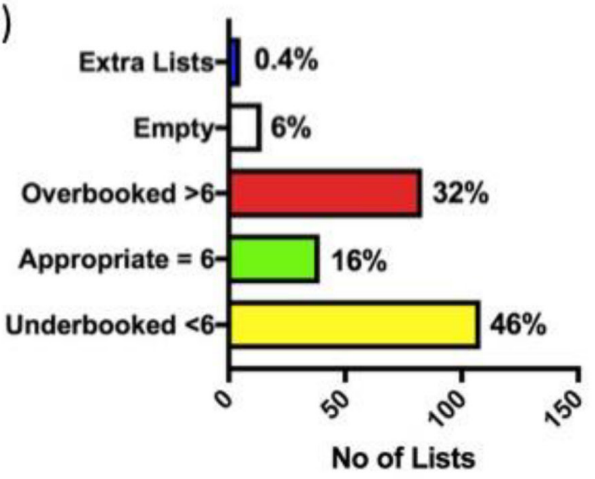

E)

Monthly Capacity vs Complexity

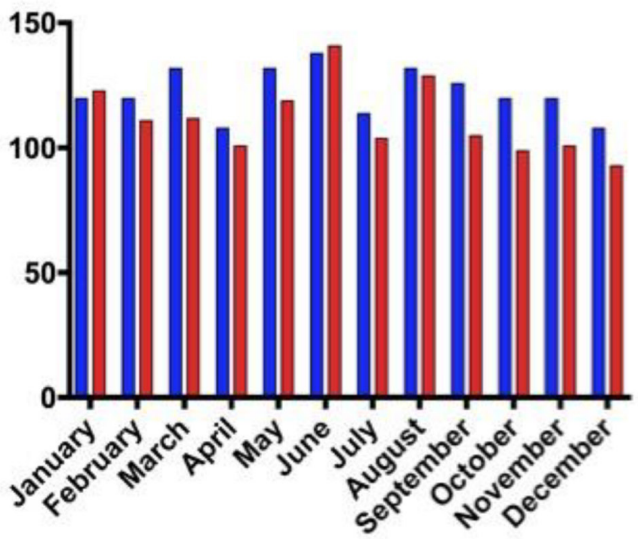

Figure 1 (A) ELCS cases and lists subdivided by ELECTIVIST risk score with average surgical and theatre times. (B) Number of ELCS lists with total ELCS list risk scores (C) ELCS lists subdivided into underbooked (score <6), appropriate (score $=6$ ), overbooked (score >6), empty and extra lists. (D) Total risk score of all ELCS cases performed in 2016 and total risk score of list capacity in 2016. (E) Total risk score of all ELCS cases performed in 2016 and total risk score of list capacity in 2016 subdivided into months. ELCS, elective caesarean section.

(figure 1C). This baseline measurement also showed that ELCS lists with a total risk score of $>6$ had a significantly higher chance of theatre over-run (relative risk (RR): 2.2) and an even higher chance of over-running with impact on emergency theatres (RR: 2.9). The capacity analysis showed that annually case complexity scores were exceeded by capacity (figure 1D) and that complexity per month exceeded capacity in 2 months. This suggested that annually no additional capacity would be required in order to accommodate the complexity of ELCS cases.

\section{Aims of the project}

The retrospective analysis allowed us to design SMART goals for the project. The first SMART goal was focused on patient safety; our specific goal was to reduce caesarean section list over-run, and over-run that impacted on emergency theatre use. Our preliminary data had shown that this was measurable, using routinely collected data. Our retrospective analysis showed that lists with a total risk score $>6$ had a significantly higher chance of over-run, suggesting this goal was attainable. The study team were 
clinically embedded within the systems that were being altered, making change within that system realistic. The time scale for this project was 9 months, with a 3-month initial implementation period and then data capture over 6 months.

The second SMART goal was focused on reducing waste; our specific goal was to reduce the number of additional lists required to meet service needs. Our preliminary data had shown that this was measurable, using routinely collected data. Our retrospective analysis showed that if lists were altered and optimised to avoid inappropriately complex or simple case mix, all caesarean sections could be done without any additional operating lists. The study team were clinically embedded within the systems that were being altered, making change within that system realistic. The time scale for this project was 9 months, with a 3-month initial implementation period and then data capture over 6 months.

\section{DESIGN AND IMPLEMENTATION}

\section{PDSA 1: design of the ELECTIVIST booking form}

The project team initially comprised an specialty trainee and two consultants in obstetrics and gynaecology with personal clinical experience of the problems caused by the current ELCS booking system. We discussed the issues contributing to ELCS list theatre over-run and decided to focus on the booking process as the factor which would be most easily improved by application of a systematic approach.

We designed a draft booking form, listing all common potential risk factors that could affect surgical complexity based on our clinical experience. Many of these were generic risk factors but some were specific to our maternity population. For example, Buckinghamshire Healthcare NHS Trust is the site of the National Spinal Injuries Centre and pregnant patients with spinal cord injuries often receive their obstetric care in our unit. These comprise a high-risk population with complex anaesthetic considerations for elective caesarean delivery but these would not be widely applicable to other units. We considered two options for the risk scoring system. Initially, we considered a Red/Amber/Green rating with each list designated to have a maximum combination of low, moderate and high risk cases. However, we felt that this could be confusing for users and therefore we sought to create a numerical system which was easier to follow.

We wanted to allocate a complexity score to each patient while simultaneously capturing all potential risk factors in the booking process. We therefore designated a scoring system from 1 to 6 with low-risk factors scoring 1 and the highest risk factors scoring 6 . These are summarised in figure 2 .

Having stratified all risk factors into categories, the final score for each case was determined by the highest scoring risk factor and not by adding the risk factor scores together. For example, a patient with fetal breech presentation with a BMI of 45 would score 1 for breech but 4 for
BMI of $>40$. The most important surgical risk factor is the BMI of 45 and therefore the final score allocated is 4 . We designated the maximum risk score for any one patient as 6 and set a standard of a maximum total score on any one ELCS list of 6 and a maximum of three cases per list. Our rationale was that this would allow a variety of permutations of case mix. For example, a list could comprise three moderate risk cases with a score of 2 or a high-risk case with a score of 4 and two low-risk cases with a score of 1 .

We sought feedback at this stage from additional stakeholders, including our local antenatal forum comprising antenatal midwives, other obstetricians and obstetric anaesthetists. Additional special features were then incorporated into the form to aid preoperative list planning, including the need for a consultant obstetrician, other surgical specialist input, preoperative anaesthetic review, antenatal corticosteroids and the requirement for cell salvage or neonatal cot availability. Using the feedback provided, we produced a final version of the booking form, which is provided in the online supplementary information A.

\section{PDSA 2: design of the ELECTIVIST booking spreadsheet}

At this stage of the project, we involved an additional specialty trainee from a different specialty with experience in IT solutions for quality improvement. Our aim was to be able to highlight inappropriately complex mix on any one list at the time of booking. All bookings onto the master Excel spreadsheet were done by antenatal clinic midwives who were not always familiar with the distinction between low and high risk surgical cases. We therefore incorporated two additional columns into the existing spreadsheet. The first column was for the individual case risk score, which was designated by the booking clinician. The second column was formatted to calculate the total risk score per ELCS list by adding the individual case scores together. If the total score for the list was 6 , the spreadsheet cell turned a green colour, indicating to staff that the list was now appropriately booked for case complexity mix and not to book additional cases on that list. If the total score for the list was $>6$, the spreadsheet cell turned a red colour, indicating to staff that the list was inappropriately complex and prompting immediate reassessment of whether the case should be moved to a different list as a result. Prior to implementation, the antenatal midwives were asked to test using the additional columns and the feedback was positive, with staff finding the system easy to use.

\section{PDSA 3: implementation of the ELECTIVIST booking form}

Our first intervention was to introduce the ELECTIVIST booking form to use in clinical practice. The project lead presented the background and aims of the project at the local academic half day departmental meeting and the form was subsequently circulated by email to all relevant staff members. Copies of the booking form were placed in antenatal clinic and incorporated into existing pre-made packs of elective caesarean paperwork. Initially there was some feedback from the surgeons that there was 


\begin{tabular}{|l|l|}
\hline Risk - LOW & \\
\hline Maternal Request & \\
\hline Previous traumatic delivery & \multirow{3}{*}{ Patient score: 1} \\
\hline Previous $3^{\text {rd }} / 4^{\text {th }}$ degree tear & \\
\hline Breech & \\
\hline Gestational Diabetes & \\
\hline Prematurity $34+0$ to 37 weeks & \\
\hline
\end{tabular}

\begin{tabular}{|c|c|}
\hline Risk - MODERATE & \\
\hline Type 1 or 2 Diabetes & \multirow{4}{*}{ Patient score: 2} \\
\hline Prematurity $28+0$ to $33+6$ weeks & \\
\hline Maternal Red Cell Antibodies requiring cross-matched blood & \\
\hline $1 \times$ previous CS & \\
\hline Small or clinically non-significant fibroids & \multirow{6}{*}{ Patient score: 3} \\
\hline Unstable or transverse lie & \\
\hline $\mathrm{BMI}>35$ & \\
\hline Tubal ligation / salpingectomy / cystectomy & \\
\hline $2 \times$ previous $\mathrm{CS}$ & \\
\hline Prematurity under $28+0$ weeks & \\
\hline
\end{tabular}

\begin{tabular}{|l|l|}
\hline Risk - HIGH & \\
\hline Multiple pregnancy (Midwifery staffing) \\
\cline { 1 - 1 } Previous midline laparotomy \\
\cline { 1 - 1 } Placenta Praevia & \\
\hline High risk of abdominal adhesions (previous notes) & \\
\hline BMI >45 & \\
\hline $3 \times$ previous CS & \\
\hline Patient declining blood products / Cell salvage & \\
\hline Spinal injury patient & \\
\hline
\end{tabular}

\begin{tabular}{|l|c|}
\hline Risk - VERY HIGH & \multirow{2}{*}{ Patient score: 5} \\
\hline BMI $>50$ & Patient score: 6 \\
\hline Placenta more previous CS & \\
\hline
\end{tabular}

Figure 2 ELECTIVIST risk factors and scoring system. BMI, body mass index; CS, caesarean section.

confusion as to whether to add all the risk scores together to allocate the final risk score. This was resolved by additional teaching of how to use the form by the project leads and the antenatal clinic midwives. At the end of this cycle, staff were confident and familiar with the correct use of the ELECTIVIST booking form.

\section{PDSA 4: implementation of the ELECTIVIST booking spreadsheet}

Our second intervention was to introduce the modified ELECTIVIST booking spreadsheet. The project lead was authorised to edit the existing master Excel spreadsheet and added the additional columns for case risk score and total list score. Antenatal clinic midwives were shown how to use the new columns and transfer all the information from the booking form onto the spreadsheet. This allowed immediate identification of overbooked lists with inappropriately complex case mix and the project leads and the antenatal clinic sister were on hand to troubleshoot in the event of any difficulty in accommodating complexity due to capacity. Initially overbooking was permitted with consultant input for the first month to allow time for staff to become familiar with the system.
PDSA 5: creation of an escalation pathway for when ELCS complexity exceeds list capacity

At 1 month following implementation, the project leads re-evaluated the booking system. Feedback from antenatal clinic staff was that at times, there were capacity issues which meant that overbooking had continued. In particular, some consultants were inclined to overbook inappropriately complex case mix without recognising the impact on the emergency obstetric theatre. By this stage of the project, other ELCS theatre staff were able to recognise when a list was overbooked due to the risk score and anticipate when a list was more likely to over-run. This could then be fed back to the original consultant in the event of theatre over-run to highlight the problem of overbooking.

This led to a new intervention of the creation of an escalation pathway. The list could be overbooked up to a score of 7 with input by any consultant who could review the cases booked and decide if appropriate. However, if the list had a total score of $>7$, then this was escalated to the antenatal lead consultant who would review existing bookings and rearrange where possible, prior to liaising 
A)

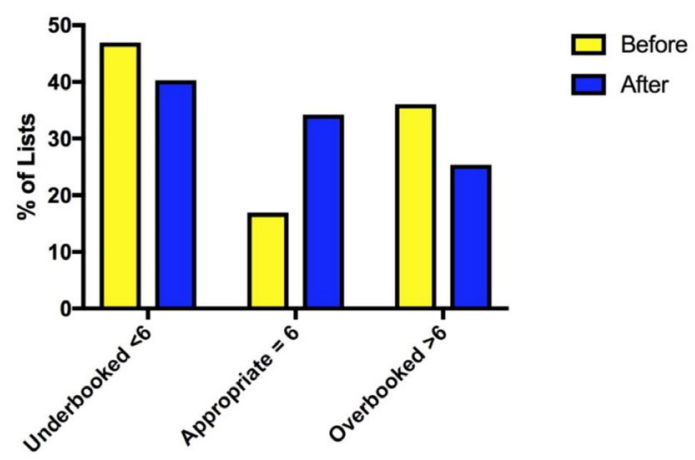

B)

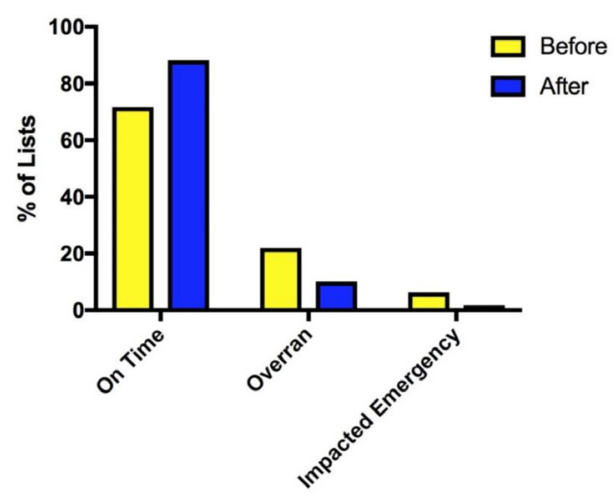

Figure 3 (A) ELCS lists subdivided into underbooked (score $<6$ ), appropriate (score=6), overbooked (score >6) before and after implementation of ELECTIVIST. (B) Percentage of ELCS lists which over-ran and impacted on emergency theatres. ELCS, elective caesarean section.

with senior managerial staff to arrange an additional ELCS list if required.

\section{RESULTS}

At 6 months after the implementation of ELECTIVIST, we compared our repeat analysis of total list risk score and theatre over-run to the initial baseline measurement. The repeat analysis data at 6 months were accurate with no missing data.

We showed that the case complexity distribution on ELCS lists had improved and there was also a significant reduction in the number of ELCS lists which over-ran, as shown in figure 3 .

The proportion of ELCS lists with over-run reduced from $21.9 \%$ to $10 \%$ and those where over-run impacted on the emergency obstetric theatre from $6.3 \%$ to $1.6 \%$. Concurrently, there was a $66 \%$ reduction in the requirement for additional ELCS lists to accommodate capacity over a 6-month period, when compared with the baseline measurement.

\section{LESSONS AND LIMITATIONS}

During this project, there were a number of positive learning points. There was excellent engagement of all stakeholders involved, from antenatal clinic midwives, obstetricians, obstetric anaesthetists, theatre staff and departmental managers. All the stakeholders recognised the ELCS list problems previously described and were highly motivated to engage in a quality improvement project that could present a solution. This meant that staff swiftly became acquainted with the ELECTIVIST risk scoring system and began to feedback to colleagues when lists were overbooked and over-ran. This aided rapid involvement of consultants at the time of booking if a list was overbooked.

We chose to modify the existing master spreadsheet meaning that antenatal clinic midwives felt comfortable with the additions, rather than having to learn a total different new system of booking. This allowed a smooth transition to the new ELECTIVIST spreadsheet and did not deter new users from engaging with it. In addition, ELECTIVIST did not require any new technological support or investment of funds which meant that the entire project was cost-neutral for the hospital. Prior to the repeat analysis, staff anecdotally were encouraged that there appeared to be considerably less over-run and if a list was appropriately booked, the entire theatre team began to recognise that the list was achievable in the time frame allocated. This meant that most of the lists that over-ran occurred in the first 3 months after implementation and that theatre efficiency continued to improve throughout.

The limitations of the project were in relation to the human interactions with the ELECTIVST system. The system was not designed to address capacity issues but to engage the booking clinicians in an immediate thought process about case mix complexity on any one list. At times when there was a capacity problem, the system had to be overseen by a designated consultant. For example, if a decision was made to perform a complex ELCS case on a particular week when there were very few remaining slots, clinicians tended to overbook the list on the basis that there was no other slot available and these lists inevitably over-ran as a result. It required a pathway for escalation to manage this more effectively and the authors acknowledge that at times, clinical need may take priority over the booking system.

We assessed for potential confounding factors which may have simultaneously contributed to the reduction in inappropriately complex case mix and theatre over-run. There was no significant difference in the grade of the operating surgeons between the baseline measurement and the repeat analysis. There were slightly fewer cases performed in the repeat analysis over a 6-month time period at 274 cases compared with 292 over the same time period in the baseline measurement in 2016. However, the average complexity score per case increased from 2.26 at baseline measurement to 2.36 in the repeat analysis. This is suggestive that the complexity of the cases was being assessed more accurately and that there was no significant difference in the number of cases performed to account for the improved ELCS list efficiency. 


\section{CONCLUSION}

The ELECTIVIST quality improvement project aimed to use a novel booking system to reduce the number of ELCS lists with inappropriately complex case mix, reduce the need for extra ELCS lists and reduce list over-run. It has been gratifyingly successful in achieving these aims and in many ways, it has exceeded the expectations of the project team.

There is little published literature regarding effective interventions to improve ELCS list performance. We suggest that ELECTIVIST is a novel and cost neutral booking system that could easily be transferred to other units. It reduces theatre over-run, particularly that impacting on emergency obstetric theatres, and thereby reduces clinical risk. The reduction demonstrated in the requirement for extra ELCS lists also represents a significant cost saving to the unit. It requires no specialist equipment or information technology and could be personalised to adapt to a different unit with a different ELCS list structure. Once set up, it requires minimal input to maintain the system and stakeholder feedback has been excellent.

Another important consideration for ELECTIVIST is that of sustainability. In the original unit, the system has remained in use with active engagement by all members of the multidisciplinary team. In order to achieve this sustainable improvement, the main stakeholders have remained flexible and welcoming of all suggestions for further improvement. At the time of publication, the authors have presented ELECTIVIST to the local maternity network and planned roll-out of the system is ongoing in two other hospitals in the region, including at a tertiary centre. Our next aim is to create a tool-kit for any hospital wishing to use this system using our experience in applying the system to other units as an individualised flexible approach is a key to the long-term sustainability of a unit incorporating ELECTIVIST into their existing structures. We also hope to incorporate the booking data into theatre checklists to create a more personalised WHO operating theatre checklist. Our longterm objective is to develop a customisable open-source IT solution to allow greater flexibility to units currently using ELECTIVIST.

Contributors KR and JC: project design, analysis and manuscript submission. RD'A: additional analysis. NK and AR: provided supervision and support during the project.

Funding The authors have not declared a specific grant for this research from any funding agency in the public, commercial or not-for-profit sectors.

Competing interests None declared.

Provenance and peer review Not commissioned; externally peer reviewed.

Open access This is an open access article distributed in accordance with the Creative Commons Attribution Non Commercial (CC BY-NC 4.0) license, which permits others to distribute, remix, adapt, build upon this work non-commercially, and license their derivative works on different terms, provided the original work is properly cited, appropriate credit is given, any changes made indicated, and the use is non-commercial. See: http://creativecommons.org/licenses/by-nc/4.0/.

\section{REFERENCES}

1. Gov.UK. NHS Maternity Statistics, England 2016 to 2017. 2017 (09 Nov 2017).

2. Wilson $\mathrm{SH}$, Fecho $\mathrm{K}$, Marshall $\mathrm{J}$, et al. Factors influencing cesarean delivery operative times: a prospective observational cohort study. Int J Obstet Anesth 2010;19:417-21.

3. Ali F, Ismail K, Johns N. Factors influencing operative times in elective caesarean sections. Eur J Obstet Gynecol Reprod Biol 2016;206:e5-e127.

4. AAGBI. OAA / AAGBI Guidelines for Obstetric Anaesthetic Services 2013. 2013.

5. Johal J, Donaldson A, Plummer J, et al. UK National Survey of Elective Caesarean Section Theatre Availability (OAA Seal of Approval No.83: Department of Anaesthesia, University Hospital of South Manchester NHS Foundation Trust.

6. Slusarczyk B. Elective Caesarean Section lists: what takes all the time? RCOG World Congress, 2013.

7. Mitchell A, Bulcock A, Alvi S, et al. Improving Caesarean Section elective theatre list time management by using an improvement science approach: Gaity Ahmad - RCOG World Congress, 2017. 